\title{
Ensino de algoritmos através de Poesia Compilada e Computação Desplugada: Relato de experiência com alunos de Ensino Fundamental
}

\author{
Soraya R. S. Medeiros ${ }^{1}$, Humberto Rabelo ${ }^{1}$, Tânia C. M. Garcia ${ }^{2}$, Isabel D. \\ Nunes $^{3}$, Felipe G. Medeiros ${ }^{4}$, Angélica F. Medeiros ${ }^{1}$, Daniele S. S. Rabelo ${ }^{5}$ \\ ${ }^{1}$ Departamento de Computação e Tecnologia - Centro de Ensino Superior do Seridó- \\ Universidade Federal do Rio Grande do Norte(UFRN)-Campus Caicó \\ ${ }^{1}$ Departamento de Educação- Centro de Ensino Superior do Seridó- Universidade \\ Federal do Rio Grande do Norte(UFRN)- Campus Caicó \\ ${ }^{3}$ Instituto Metrópole Digital - Universidade Federal do Rio Grande do Norte (UFRN) \\ ${ }^{4}$ Instituto Federal do Rio Grande do Norte(IFRN)- Campus Caicó \\ ${ }^{5}$ Secretaria de Educação à Distância (SEDIS) Universidade Federal do Rio Grande do \\ Norte (UFRN) - Natal - RN - Brasil \\ \{soraya.roberta.js, hrabeloufrn,fgmfrioforte, rabrlodanni, angelicafelixx \}@gmail.com, \\ tania_cristina2005@yahoo.com.br,bel@imd.ufrn.br
}

\begin{abstract}
Computational Thinking (CT) is a skill that must be learn still in childhood, because it will be applied along life. Algorithms Teaching is one approach for teaching CT, however not all schools have informatics labs. This work aims to report an application experience of CS Unplugged in Algorithms Teaching for a public school sixth grade class, using poem text genre and Python programming language. For doing that, students joined workshops that propitiated analogies between poems and algorithms, resulting in learning of common concepts to both areas in a playful way.

Resumo. Pensamento Computacional $(P C)$ é uma habilidade que deve ser aprendida ainda na infância, pois será aplicado ao longo da vida do ser humano. $O$ ensino de algoritmos é uma abordagem utilizada para ensinar o $P C$, entretanto, nem todas as escolas dispõem de laboratórios de informática. Este trabalho visa relatar uma experiência de aplicação de Computação Desplugada no ensino de algoritmos em uma turma do $6^{\circ}$ ano de uma escola da rede pública, com a utilização do gênero textual poema e a linguagem de programação Python. Para que fosse possível, os alunos participaram de oficinas que propiciaram analogias entre poemas e algoritmos, resultando no aprendizado dos conceitos comuns às duas áreas de forma lúdica.
\end{abstract}


VII Congresso Brasileiro de Informática na Educação (CBIE 2018)

Anais do XXIV Workshop de Informática na Escola (WIE 2018)

\section{Introdução}

O Pensamento Computacional (PC) é um conjunto de técnicas que utiliza conceitos da Computação para resolver problemas de diversas áreas, sendo uma habilidade fundamental que deve ser desenvolvida ainda na infância, assim como ler, escrever ou efetuar cálculos básicos, pois será aplicado ao longo da vida do ser humano [Wing 2006]. Em um trabalho de revisão recente sobre aplicação de Computação no Ensino Básico brasileiro, a área de algoritmos e programação tem despontado como uma das principais estratégias pesquisadas para o desenvolvimento de PC em crianças e adolescentes, assim como Computação Desplugada e Jogos Digitais [Bordini et al. 2016].

Tradicionalmente, o ensino de algoritmos e a adoção de jogos digitais para o desenvolvimento do PC requerem ferramentas que necessitam do uso do computador para sua aplicação, e, apesar dos esforços de professores e gestores, as escolas nem sempre dispõem de boa estrutura física de laboratórios de informática, seja pela quantidade insuficiente de equipamentos, pela velocidade de conexão à internet, ou ainda pela permanência de máquina com defeito, conforme aponta pesquisa realizada recentemente [Brasil 2017]. Diante desse contexto, surge a necessidade de novas metodologias que não dependam do uso de software e hardware, tais como Computação Desplugada [Santos et al. 2015]que consiste em ensinar o pensamento computacional sem fazer uso do computador.

O presente estudo, neste sentido, propõe relatar uma experiência de aplicação de Computação Desplugada em ensino de algoritmos para desenvolvimento de PC em uma turma do $6^{\circ}$ ano de uma escola da rede pública, com a utilização da linguagem de programação Python, tendo como base um estudo de caso recente [Andrade, Medeiros and Medeiros 2017] que utiliza o Manifesto Literário Poesia Compilada como ferramenta para criar um ambiente interdisciplinar entre as disciplinas de Língua Portuguesa e Algoritmos. Como resultado, os alunos mostraram melhor desempenho na interpretação de textos, em específico do gênero textual poema, começaram a participar e a interagir nas aulas, tiveram maior rendimento acadêmico e menos indisciplina em sala de aula.

Este trabalho está organizado em cinco seções. A seção dois aborda o PC no ensino básico. A seção três apresenta um panorama dos trabalhos que utilizam Computação Desplugada para ensinar algoritmos, tendo como público-alvo crianças nas séries iniciais do ensino básico. A quarta seção discorre sobre como este trabalho combinou Computação Desplugada e conceitos do Manifesto Literário Poesia Compilada para ensinar algoritmos. A quinta, por sua vez, diz respeito à aplicação da metodologia proposta. A sexta seção relata os resultados obtidos focalizando o que os alunos conseguiram compreender com a oficina, seguida de considerações finais.

\section{Pensamento Computacional no Ensino Básico}

De acordo com [Blikstein 2008], o PC não se trata de saber navegar na internet, enviar e-mail ou operar um processador de texto, porém saber usar o computador como um instrumento de aumento do poder cognitivo e operacional humano, tal como o aumento da produtividade, inventividade e criatividade. $\mathrm{O}$ autor divide o PC em duas etapas, 
VII Congresso Brasileiro de Informática na Educação (CBIE 2018)

Anais do XXIV Workshop de Informática na Escola (WIE 2018)

sendo a primeira delas o "pensar computacionalmente", pela qual é possível identificar as tarefas cognitivas que podem ser feitas de forma mais rápida e eficiente por um computador, e a segunda, em saber programar essa máquina para realizar essas tarefas cognitivas.

Para que se possa realizar esta segunda etapa é necessário que se aprenda como fazer um algoritmo, decorrendo a importância de ensiná-lo cada vez mais cedo nas escolas, a fim de que ele possa contribuir para desenvolver o PC citado por [Wing 2006] e reforçado por [Blikstein 2008] por ser uma das abordagens para se ensinar PC. Além disso, [Ray et al. 2011] definem PC como um processo de resolução de problemas que inclui, dentre outros pontos, a solução automática por meio de pensamento algorítmico.

Atualmente, no Brasil, existem algumas problemáticas para se trabalhar o PC em sala de aula, uma delas é a adoção curricular, sobre a qual, na atual proposta da Base Nacional Comum Curricular (BNCC) para o Ensino Básico, o PC está expresso e motivado de forma estrita, tendo sido emitido um Parecer da SBC sobre este posicionamento, ressaltando a importância das competências e habilidades do Ensino de Computação na Educação Básica [Leite and Silva 2017]. [França and Tedesco 2015] enfatizam a necessidade de discutir a definição de diretrizes curriculares para o ensino da habilidade de PC nas escolas brasileiras. Os autores ressaltam que o ensino do PC não deve cobrir apenas a manipulação de recursos digitais, mas os fundamentos da Computação enquanto ciência.

Além de o currículo ser um desafio, [França and Tedesco 2015] elucidam a formação de professores e a forma como poderiam estar sendo ensinados e aprendidos algoritmos e lógica de programação. Sobre este último, comentam que é recorrente o uso de ambientes visuais de programação tais como Scratch ${ }^{1}$, App Inventor ${ }^{2}$, AgentSheets ${ }^{3}$ e RoboMind ${ }^{4}$. Levando em consideração a relevância do ensino de algoritmos para se trabalhar com PC nas escolas, uma vez que é comum a problemática física dos laboratórios de informática, bem como os desafios metodológicos para se implantar e trabalhar com as estratégias do PC nas escolas públicas brasileiras, surge a necessidade de se utilizar um meio alternativo frente a essas demandas, nesse caso, a Computação Desplugada.

\section{Ensino de Algoritmos com Computação Desplugada no Ensino Básico}

O termo Computação Desplugada, do inglês, Computer Science Unplugged, foi descrito por [Bell, Whitten and Fellows 2007], e consiste em atividades práticas que visam ensinar conceitos da Computação por meio de atividades manuais e lúdicas, sem demasia de detalhes técnicos e distrações, por não necessitar de um software e hardware. Diversos estudos têm mostrado a relevância da Computação Desplugada para a difusão das diferentes estratégias do PC, por permitir que exista adequação para distintas estruturas escolares, visto que não requer o uso de software e hardware, bem como desmistifica a ideia de que o computador resolve problemas sem intervenção humana [Vieira, Passos and Barreto 2013].

\footnotetext{
www.scratch.mit.edu/

www.appinventor.mit.edu

www.agentsheets.com/

www.robomind.net/pt
} 
VII Congresso Brasileiro de Informática na Educação (CBIE 2018)

Anais do XXIV Workshop de Informática na Escola (WIE 2018)

O ensino de algoritmos pode ser desenvolvido por meio do uso de ferramentas, tais como o Scratch [Silva, Souza and Silva 2016], ou por meio de Computação Desplugada. [Santos et al. 2015] abordam a utilização de Computação Desplugada para alunos do $5^{\circ}$ ano do Ensino Fundamental para promover habilidades do $\mathrm{PC}$ através do ensino de algoritmos. O estudo denominado "Algoritmos Desplugados" teve inspiração na ferramenta Scratch e faz a utilização de blocos de borracha de diferentes tamanhos, em que cada bloco é encaixado em outro e um conjunto de blocos devidamente encaixados formam um algoritmo. Para a execução dos algoritmos são utilizados diversos tabuleiros impressos em folhas de tamanho A4, além de itens coletáveis e desafios.

[Silva et al. 2017] relataram a experiência de uma oficina ao introduzir a Computação na Educação Básica e a familiarização com os conceitos de algoritmos e de programação mediante exemplos práticos, como atravessar a rua e trocar uma lâmpada. A oficina foi realizada em uma escola pública de ensino fundamental, em uma turma do $8^{\circ}$ e $9^{\circ}$ ano que não dispunha de laboratório de informática em aulas de Língua Portuguesa e Matemática. Na primeira, foram utilizadas questões de semântica (interpretação de texto, sentido das palavras e expressões no contexto). Já na segunda, foram apresentadas expressões numéricas simples e jogo de sinal. Como resultado, notou-se nos alunos uma mudança positiva quanto ao raciocínio e a capacidade de solucionar problemas, além do aumento do interesse e curiosidade para as áreas relacionadas à Computação.

[Schulz. et al. 2017] discutem a experiência que envolveu Computação Desplugada e o uso do Tangram para ensinar algoritmos a uma turma do $5^{\circ}$ ano do Ensino Fundamental de escola pública do município de Santa Cruz do Sul - RS. Antes de iniciar o estudo dos algoritmos, os pilares do Pensamento Computacional foram trabalhados com os discentes, possibilitando a identificação de diferentes caminhos e quais soluções seriam mais eficientes para a resolução de um problema.

Tal como em [Santos et al. 2015], este trabalho faz uso de Computação Desplugada para ensinar algoritmos para crianças utilizando sequências lógicas, condicional e abstrações, entretanto, não adota o $5^{\circ}$ ano e não se inspira em Scratch, por escolha dos autores. A escolha quanto ao gênero textual poema se dar porque é um conteúdo que os alunos já estão conhecendo e conseguem fazer analogias mais facilmente, principalmente no $6^{\circ}$ ano. Assim como [Silva et al. 2017], esta experiência utiliza as aulas de Língua Portuguesa para difundir o ensino de algoritmos, no entanto, não o faz em aulas de Matemática, pois busca se diferenciar da maior parte dos trabalhos que associam algoritmos sempre aos números e consequentemente ao cálculo.

Ao invés de trabalhar com o Tangram, como fez [Schulz et al.2017], é utilizada a linguagem de programação Python para introduzir os conceitos de algoritmos e lógica de programação por meio do quadro branco, cadernos ou blocos de anotações dos próprios alunos. O diferencial deste estudo, frente aos demais, é empregar o gênero textual poema para trabalhar com o ensino de algoritmos em uma turma do $6^{\circ}$ ano do Ensino Básico, nas aulas de Língua Portuguesa e Literatura, usando a linguagem de programação Python através de Computação Desplugada. 
VII Congresso Brasileiro de Informática na Educação (CBIE 2018)

Anais do XXIV Workshop de Informática na Escola (WIE 2018)

\section{Poesia Compilada}

Poesia Compilada ${ }^{5}$ é um Manifesto Literário que propõe compilar as sintaxes e semânticas do gênero textual poema com as de um algoritmo, tendo sido criado por Soraya Roberta dos Santos Medeiros quando aluna do Instituto Federal do Rio Grande do Norte (IFRN) em 2012. O primeiro relato do uso do gênero textual poema para ensinar algoritmos está presente no estudo de [Andrade, Medeiros and Medeiros 2017], o qual aborda a utilização do editor de textos Poesia() ${ }^{6}$ para o ensino de Algoritmos em turma de alunos ingressantes do Bacharelado em Sistemas de Informação com base no Manifesto Literário Poesia Compilada.

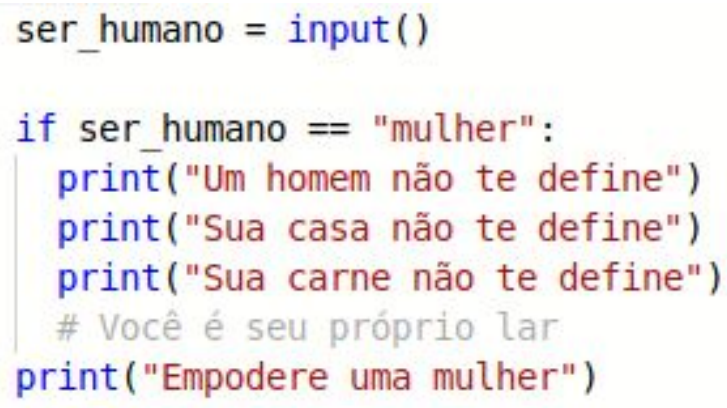

Figura 1. Exemplo de Poesia Compilada. Fonte: facebook/poesia compilada

A Figura 1 retrata uma Poesia Compilada escrita na linguagem de programação Python, utilizando a letra da música "Triste, Louca ou Má” da banda Francisco El Hombre. Essa poesia retrata a entrada de dados através da função input, o processamento, explícito por meio da estrutura condicional e a saída dos dados se dá por meio da função print. A partir de uma breve revisão fundamentada no aporte teórico da área da psicologia da aprendizagem, percebe-se que o Poesia Compilada, ao apoiar-se nas bases conceituais da psicologia cognitiva do sociointeracionismo vigotskiano, no que se refere à Zona de Desenvolvimento Proximal - ZDP, apontada por [Vygotsky 1984], possibilita ao aluno o desenvolvimento potencial de algoritmos. Para tanto, faz-se necessário que sejam levados em consideração aspectos relativos ao desenvolvimento real com foco na mediação adulto/criança. Isso porque, para a teoria vigotskyana, a ZDP concentra funções que ainda serão amadurecidas, mas que já se encontram de modo embrionário, tal como o que se percebe neste estudo. Ademais, o trabalho faz uso do conceito de interdisciplinaridade, discutido por [Piaget 1972], na medida em que relaciona os conceitos entre os algoritmos e o gênero textual poema, contribuindo para que exista cooperação entre as duas áreas e a geração de enriquecimento mútuo.

\section{Como foram desenvolvidas as oficinas}

A estratégia adotada para aplicar os conceitos do Manifesto no ensino de algoritmos consistiu em realizar oficinas sobre o tema e analisar aspectos relacionados à satisfação e motivação do público discente em aprender Algoritmos com Poema. As oficinas aconteceram no período de 24 de abril a 22 de maio de 2018, na Escola Municipal Professora Maria de Lourdes de Medeiros, na cidade de Jardim do Seridó, Rio Grande

www.poesiacompilada.com

www.poesiacompilada.com/editor 
VII Congresso Brasileiro de Informática na Educação (CBIE 2018)

Anais do XXIV Workshop de Informática na Escola (WIE 2018)

do Norte(RN) tendo como público-alvo alunos do $6^{\circ}$ ano, totalizando 25 discentes, sendo desenvolvida por Soraya Roberta, aluna do curso Bacharelado em Sistemas de Informação (BSI). A oficina dividiu-se em 2 etapas: 1) conhecer a escola e planejar as oficinas; 2) aplicar as oficinas.

Para que se cumprisse a etapa 1, foi efetuada uma visita prévia em que se conversou com a professora de Língua Portuguesa e literatura das duas escolas. Nesse encontro, identificou-se que só seriam possíveis realizar 4 encontros, sendo 1 por semana, pois existiam poucas aulas para as docentes trabalharem os demais conteúdos com os alunos. Além disso, verificou-se que a escola possuia laboratório de informática, porém não se encontravam aptos para uso por estarem sucateados, resultando na necessidade de se aplicar Computação Desplugada. Outro fator observado e determinante para realizar as oficinas a partir da utilização do gênero textual poema foi que os alunos estavam aprendendo a estrutura desse gênero à época.

Para a realização da etapa 2 foi necessária a utilização de um projetor, dos próprios cadernos dos alunos, pincéis de quadro branco e o quadro branco. Na primeira semana, a orientadora da oficina relembrou aos alunos por meio de poemas como é formada a estrutura deste gênero, tal como as diferenças entre versos e estrofes, poema e poesia, dentre outros elementos. O exercício inicial consistiu na elaboração de poemas pelos alunos, recitação para a turma, bem como explicar cada elemento que tinham utilizado. Como resultado, cada aluno produziu seu poema em seu respectivo caderno e recitou para a turma explicando o que tinha em cada poema, apontando as diferenças frente às produções dos demais colegas.

$\mathrm{Na}$ segunda semana, os alunos realizaram a tarefa "Tinha um algoritmo no meio do caminho". Sendo divididos em grupos, construíram poemas que seguissem o trajeto de ir até à escola, recitando-os, em seguida, para a turma. Nesta aula foram trabalhados os conceitos de abstração, sequência lógica e condicional. Para efetuar este trabalho, os alunos se dividiram em grupos de até três, de acordo com a afinidade em sala de aula, e tinham que abstrair, detalhar o passo a passo escrevendo em formato de um poema, onde cada verso era um passo para ir ou até à escola. Antes dos alunos começarem, o orientador expôs o algoritmo dele para ir de casa até à escola aplicar a oficina, como forma de exemplificar o que era proposto. Na apresentação para a turma, eles tinham que recitar o poema, em seguida dizer o que tinha de diferente ou repetido nos poemas dos colegas.

$\mathrm{Na}$ terceira semana, o orientador informou que todos aqueles passos poderiam ser feitos utilizando uma outra linguagem que os alunos ainda não tinham aprendido, bem como cada poema poderia ser representado de uma única forma, mudando apenas a quantidade de vezes que um verso ou estrofe poderia ser repetido. Por fim, foi compartilhado que todas aquelas sequências finitas de passos lógicos escritas em formato de poema, tratavam-se de um algoritmo, explicando que um algoritmo pode ser escrito em uma linguagem de programação, tal como a linguagem Python, que tem em sua estrutura diversas funções, como a função print que pode exibir, dentre outros conteúdos, um poema.

Nesta aula os alunos não realizaram nenhuma atividade e, apesar da postura passiva, houve interação. Por fim, na quarta semana, foi proposta aos discentes a atividade E agora, José?, pela qual eles tinham que construir um poema utilizando a linguagem de programação Python e a função print, bem como recitarem 
VII Congresso Brasileiro de Informática na Educação (CBIE 2018)

Anais do XXIV Workshop de Informática na Escola (WIE 2018)

individualmente para toda a turma. Para realizá-la, cada aluno fez seu próprio poema utilizando seu caderno e, em seguida, recitou para toda a turma e explicou o que tinha aprendido com a atividade.

Considerando que não se tinha muito tempo para mostrar os demais elementos de um algoritmo, tais como laço de repetição em Python, propôs-se aos alunos, para a aula seguinte, a elaboração de um algoritmo utilizando a função print a partir de algum poema, que fosse feito por eles ou por algum poeta conhecido. A avaliação das atividades levou em conta a produção em sala de aula, o quão próximo de um algoritmo executável os poemas dos alunos chegaram, e a frequência nas oficinas uma análise docente sobre o resultado do bimestre em que foram aplicadas as oficinas, assim como em [Silva et al. 2017].

\section{Resultados e discussões}

Nesta seção, apresenta-se os resultados observados e as considerações tecidas a partir dos mesmos.

\subsection{Atividades 2 e 3}

Neste algoritmo da Figura 2 (a), o discente estruturou em uma estrofe e dez versos o poema, bem como utilizou o condicional "se, se nao" para expressar a condição e a sequência lógica. As rimas não eram algo obrigatório, visto que um poema pode ou não ter rimas. Ao longo das análises, percebeu-se que os alunos tiveram bastante dificuldade em apresentar para a turma a sua produção, visto que alguns deles não apresentaram por causa da timidez.

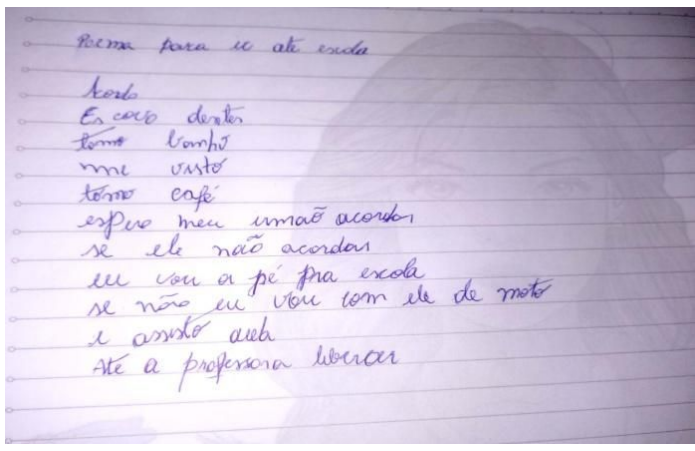

(a)

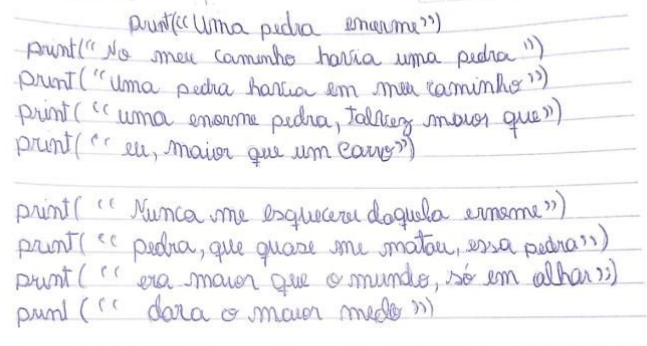

(b)

Figure 2. Produção de um algoritmo por um dos alunos. Fonte: Autores.

$\mathrm{Na}$ Figura 2 (b), encontra-se uma produção referente à atividade “E agora, José?", onde o aluno fez uso de características sintáticas e semânticas de algoritmos e do gênero textual poema para criar um poema por meio da função print da linguagem de programação Python. No que concerne à semântica e sintaxe, ele conseguiu compreender e fez uso de intertextualidade por meio do poema "No meio do caminho tinha uma pedra", do Carlos Drummond de Andrade. 
VII Congresso Brasileiro de Informática na Educação (CBIE 2018)

Anais do XXIV Workshop de Informática na Escola (WIE 2018)

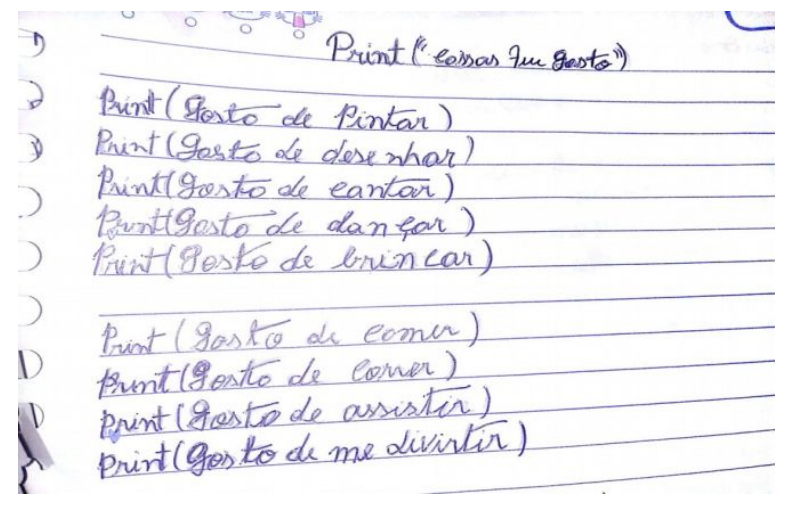

Figure 3. Produção de um algoritmo por um dos alunos. Fonte: Autores.

Na Figura 3, o aluno confundiu-se com a sintaxe da linguagem de programação ao trocar as posições. Tais erros poderiam ser melhor trabalhados se os alunos tivessem acesso a um Ambiente de Desenvolvimento Integrado, do inglês "integrated development environment"-IDE, pois a presença do que seria o erro de sintaxe ficaria mais evidente, e eles poderiam corrigir a tempo de finalizar os algoritmos. Para os alunos, a oficina foi satisfatória, sendo isto evidenciado pelo interesse e participação, o que foi evidenciado pela solicitação de continuidade das atividades desenvolvidas. Verifica-se, a partir das produções dos alunos, que eles conseguiram assimilar os conceitos aprendidos sobre algoritmos, uma vez que eles apresentam características de poemas, tais como a intertextualidade, versos e estrofes, e levaram à compreensão sobre o que são algoritmos e como são construídos, por meio da correta aplicação da função print, o que pode ser corroborado pelo pensamento de [Vygotski 1984] e [Piaget 1972], ao existir a interdisciplinaridade entre os conceitos do gênero textual poema e algoritmos.

\subsection{Relato sobre a Análise de Aprendizagem}

A professora de Português relatou que os alunos não participavam das aulas e apresentavam péssimo comportamento e rendimento acadêmico, tal como a interpretação dos textos, em específico o gênero textual Poema. Ao final do $3^{\circ}$ bimestre, quando foram aplicadas as oficinas, houve uma melhora na participação das aulas e desempenho da interpretação de texto, visto que as oficinas fizeram com que os alunos ao pegarem um texto/poema, pudessem primeiro pensar sobre o que ele abordava, para só depois começar a desenvolver um posicionamento sobre sua proposta, indo ao encontro do conceito de PC elucidado por [Blinkstein 2008].

\section{Considerações finais}

Neste trabalho foi possível observar a aplicação do ensino de algoritmos e programação, uma das estratégias para se trabalhar com o PC, em uma escola pública que possuía laboratório de informática não funcional. Para contornar tal problemática, utilizou-se Computação Desplugada. O diferencial inovador deste trabalho frente às literaturas revisadas, é utilizar o gênero textual poema para ensinar algoritmos com a linguagem de programação Python, fazendo com que os alunos refletissem sobre o seu cotidiano por meio de poemas, e pudessem expressá-los em forma de um algoritmo. 
VII Congresso Brasileiro de Informática na Educação (CBIE 2018)

Anais do XXIV Workshop de Informática na Escola (WIE 2018)

Compartilhar PC de forma lúdica e fazer analogias com conteúdos em que os alunos estão trabalhando ainda no Ensino Básico estimula a disseminação e desmistificação sobre o que é Computação e como ela está presente em diversos lugares e áreas da Sociedade, tal como a área de Literatura. Possibilita que os alunos consigam interpretar melhor textos/ poemas, bem como suscita conhecimentos pré-concebidos, como foi o caso do gênero textual poema, uma vez que se pode aplicar em novos conhecimentos, como os algoritmos.

Além disso, propor aos alunos que apresentem/recitem seus poemas/algoritmos, façam trabalhos em grupos e proponham soluções para problemas, contribui para que eles possam ampliar sua visão de mundo e serem sujeitos mais ativos e transformadores. Por fim, para trabalhos futuros, sugere-se a aplicação mais detalhada dos demais conceitos sobre algoritmos, bem como a comparação entre turmas com o ensino tradicional e com o ensino com Poesia Compilada.

\section{Referências}

Andrade, A. P. V., Medeiros, I. G., Medeiros, S. R. S. (2017). Ensino de algoritmos com Poesia Compilada: experiências em turmas iniciais no Bacharelado em Sistemas de Informação. In II Congresso sobre Tecnologias na Educação, p. 523-529.

Bell, T.; Witten, I. e Fellows, M. (2011). "Computer Science Unplugged - Ensinando Ciência da Computação sem o uso do Computador". Tradução de Luciano Porto Barreto, 2011. Disponível em: http://csunplugged.org/. Acesso em 02/02/2018.

Bordini, A., Avila, C. M. O., Weisshahn, Y., Cunha, M. M., Cavalheiro, S. A. C. , Foss, L. , Aguiar, M. S. , Reiser, R. H. S. (2016). Computação na Educação Básica no Brasil: o Estado da Arte. Revista de Informática Teórica e Aplicada , v. 23, n. 2, p. 210-238.

Brasil. (2017). INFRAESTRUTURA: Equipamentos, internet e condições gerais. https://www.todospelaeducacao.org.br/tecnologia/equipamentos-tecnologia-escola/ [acesso em 20 de Abril].

França, R. and Tedesco, P. (2015). Desafios e Oportunidades de Ensino na Computação Básica na Educação Básica no Brasil, in Anais dos Workshops do IV Congresso Brasileiro de Informática na Educação (CBIE 2015).

Leite, M. and Silva, S.R.F. Da (2017). Redimensionamento da Computação em Processo de Ensino na Educação Básica: O Pensamento Computacional, o universo e a Cultura Digital", in Anais do dos Workshops do VI Congresso Brasileiro de Informática na Educação (WCBIE 2017).

Matemática no Ensino Fundamental - Anos iniciais: Unidades Temáticas, Objetivos de Conhecimento $\mathrm{e}$ Habilidades. http://basenacionalcomum.mec.gov.br/abase/\#fundamental/matematica-no-ensino-fu ndamental-anos-iniciais-unidades-tematicas-objetos-de-conhecimento-e-habilidades, [acessado em 11 de Abril de 2018].

$\mathrm{O}$

Pensamento

Computacional

$([\mathrm{Sd}])$. 
VII Congresso Brasileiro de Informática na Educação (CBIE 2018)

Anais do XXIV Workshop de Informática na Escola (WIE 2018)

http://www.blikstein.com/paulo/documents/online/ol_pensamento_computacional.ht $\mathrm{ml}$, [acesso em 20 de Abril].

Piaget, J. Epistemologie des relations interdisciplinaires. In: CERI (Ed.). L'interdisciplinarité: problèmes d'enseignement et de recherche dans les Universités. Paris: UNESCO/OCDE, 1972.

Ray, L., Whitehead, C., Shamim, K., Summers, W., \& Obando, R. (2011). School Level Computer Science Education and Computer Science Teacher Training in the US: An Overview and an Example Solution. In Society for Information Technology \& Teacher Education International Conference (Vol. 2011, No. 1, pp. 3931-3938).

Santos, G., Silva, W., Cavalheiro, S., Foss, L., Aguiar, M. S. , Pernas, A. M. , Bois, A. , Reiser, R (2015). Proposta de atividade para o ensino fundamental do ensino: Algoritmos Desplugados. "Relato sobre o ensino de algoritmos a estudantes do quinto ano do ensino público fundamental," in Anais do XXI Workshop de Informática na Escola (WIE).

Schulz, JM, Queiroz, BTS, Frantz, J. e Cruz, MEJK Da (2017). Computação Desplugada e uso do Tangram no Ensino de Algoritmos. Anais do Salão de Ensino e de Extensão, v. 0, n. 0, p. 351.

Silva, G.T. Da, Souza, J.L. De e Silva, L.A.M. Da (2016). Ferramenta Scratch para o Aprendizado de Programação no Ensino Fundamental I", in Anais dos Workshops do V Congresso Brasileiro de Informática na Educação (CBIE).

Silva, N. C., Lima, A. C., de Souza, N., \& de Sousa, D. F. (2017). Raciocínio Lógico nas Escolas: Uma Introdução ao Ensino de Algoritmos de Programação. In Anais dos Workshops do Congresso Brasileiro de Informática na Educação (Vol. 6, No. 1, p. 1011).

Vygotsky, L. S. (1984). A formação social da mente. Martins Fontes.

Vieira, A. Passos, O. and Barreto, R. (2013). Um Relato de Experiência do Uso da Técnica Computação Desplugada, in Anais de Workshop sobre Educação em Computação(WEI).

Wing, J. M. (2006)“Computational thinking," Communications of the ACM, vol. 49, pp. 33-35. 